\title{
Unbiased Atlas Formation Via Large Deformations Metric Mapping
}

\author{
Peter Lorenzen ${ }^{1}$, Brad Davis ${ }^{1}$, and Sarang Joshi ${ }^{1,2}$ \\ 1 Department of Computer Science \\ 2 Department of Radiation Oncology, University of North Carolina, \\ Chapel Hill, NC 27599, USA \\ \{lorenzen, davisb, joshi\}@cs.unc.edu
}

\begin{abstract}
The construction of population atlases is a key issue in medical image analysis, and particularly in brain mapping. Large sets of images are mapped into a common coordinate system to study intrapopulation variability and inter-population differences, to provide voxelwise mapping of functional sites, and to facilitate tissue and object segmentation via registration of anatomical labels. We formulate the unbiased atlas construction problem as a Fréchet mean estimation in the space of diffeomorphisms via large deformations metric mapping. A novel method for computing constant speed velocity fields and an analysis of atlas stability and robustness using entropy are presented. We address the question: how many images are required to build a stable brain atlas?
\end{abstract}

Keywords: Computational anatomy; Brain Atlases; Image Metric Space.

\section{Introduction}

Computational anatomy is the study of anatomical variation [1]. For a set of images representing a population, a natural problem in computational anatomy is the construction of an atlas — an image that serves as a representative for the population. Such an atlas must represent the anatomical variation present in the image population [2]. A major focus of computational anatomy has been the development of image mapping algorithms 3456] that map and transform a single brain atlas onto a population.

In the recent and related work of [7], the authors developed a large deformation template estimation algorithm by averaging velocity fields. Most other previous work 89. in atlas formation has focused on the small deformation setting in which arithmetic averaging of displacement fields in well defined. We do not make this small deformation assumption.

To generate the deformations for producing atlases, we apply the theory of large deformation diffeomorphisms [104]. We simultaneously estimate the unbiased atlas and the transformations which map the atlas to each population image. Linear averaging cannot be applied directly to the large deformation setting as, under the large deformation model, the space of transformations is not a vector space, but rather the infinite dimensional group $\mathcal{H}$ of diffeomorphisms 
of an underlying coordinate system $\Omega$. In our previous work [11, we address this problem by posing anatomical atlas creation as a statistical estimation problem where the notion of simple intensity averaging is extended to general metric spaces first proposed by Fréchet [12]. In [11], we developed a method for unbiased construction of atlases based on an iterative greedy method for generating large deformation diffeomorphisms.

In this paper, we present a complete large deformations metric mapping (LDMM) methodology introduced by [13. Both the greedy and LDMM implementations provide large deformation coordinate system transformations. In [11, the solution to the atlas formation problem generates paths through the space of diffeomorphisms, the length of which cannot be used to define a metric as the method provides a locally optimal rather than full space-time solution. In contrast, the variational optimization of the atlas formation cost function in the LDMM algorithm, gives geodesic paths on the manifold of diffeomorphic transformations, $\mathcal{G}$, the lengths of which places the orbit of transformed images into a metric space. In this way, we build a geodesic atlas.

We use the entropy of voxel intensities to measure the robustness and stability of unbiased atlases. In the context of in MR images, entropy is often used to assess the degree to which an image differs from an ideal where an ideal image intensity histogram consists of a small number of modes representing tissue classes [1415. We study the stability of atlases produced by our method by building atlases, of increasing population size, using multiple permutations of images from a database of images.

The remainder of this paper is organized as follows: in Section 2, the unbiased atlas formation problem is developed; in Section 3, the Euler-Lagrange equations used to characterize the LDMM and implementation details and a novel method for constant velocity computation are presented; and in Section 4, an analysis of the atlas stability and robustness using entropy are reported.

\section{Method}

In our previous work [11, we exemplify the atlas estimation problem by first considering a population of $N$ images $\left\{I_{i}\right\}_{i=1 \ldots N}$ acquired by the same imaging modality which have been rigidly aligned. We seek the representative image, $\hat{I}$, that requires the minimum amount of energy to deform into each population image $I_{i}$. In the spirit of 13 , we define dense transformations, from the infinite dimensional group of diffeomorphisms $\mathcal{H}$, and fluid flow vector fields in the following manner: the group elements of the change of coordinates $\varphi_{i}$ such that $\hat{I}=\varphi_{i}^{-1} I_{i}=I_{i} \circ \varphi_{i}$ are generated as the end-points $\varphi_{i}=\phi_{i}(1)$ of the flows of time-dependent vector fields $v_{i}(\cdot, t), t \in[0,1]$ from the space of smooth vector fields $V$ via

$$
\dot{\phi}_{i}^{v}(t)=\frac{d}{d t} \phi_{i}^{v}(t)=v_{i}\left(\phi_{i}^{v}, t\right)
$$

where the superscript $v$ in $\phi_{i}^{v}$ is used to explicitly denote the dependence of $\phi_{i}$ on the associated velocity field $v$. The terminal point of the curve $\phi_{i}^{v}$ at $t=0$ is 
$\phi_{i}^{v}(0)=e \in \mathcal{G}$ where $e$ is the identity transformation $e(x)=x, \forall x \in \Omega$. The end point of the curve $\phi_{i}^{v}$ at $t=1$ is the particular diffeomorphism $\phi_{i}^{v}(1)=\varphi_{i} \in \mathcal{G}$ that links the images $\hat{I}$ and $I_{i}$ such that $\hat{I}=\varphi_{i}^{-1} I_{i}=I_{i} \circ \varphi_{i}$. The transformations $\varphi_{i}$ are generated by integrating velocity fields, $v_{i}$, forward in time.

Given $\mathcal{H}$, with associated metric $D: \mathcal{H} \times \mathcal{H} \rightarrow \mathbb{R}$, along with an image dissimilarity measure $E\left(I_{1}, I_{2}\right)$, we wish to find the image $\hat{I}$ such that

$$
\left\{\hat{\varphi}_{i}, \hat{I}\right\}=\underset{\varphi_{i} \in \mathcal{H}, I}{\operatorname{argmin}} \sum_{i=1}^{N}\left(E^{2}\left(I, I_{i} \circ \varphi_{i}\right)+D^{2}\left(e, \varphi_{i}\right)\right) .
$$

We induce a metric on $\mathcal{H}$ by a Sobolev norm via a partial differential operator $L$ on the velocity fields. Let $\varphi$ be a diffeomorphism isotopic to the identity transformation $e$, that is, there exists a continuous family of diffeomorphisms from $e$ to $\phi$. We define the squared distance $D^{2}(e, \varphi)$ on the space $V$ of smooth velocity vector fields on the domain $\Omega$, as

$$
D^{2}(e, \varphi)=\min _{v: \dot{\phi}(t)=v\left(\phi^{v}, t\right)} \int_{0}^{1}\|L v(t)\|_{V}^{2} d t .
$$

The distance between any two diffeomorphisms is defined by

$$
D\left(\varphi_{1}, \varphi_{2}\right)=D\left(e, \varphi_{1}^{-1} \circ \varphi_{2}\right) .
$$

This distance satisfies all the properties of a metric [16].

Having defined a metric on $\mathcal{H}$, the minimum energy template estimation problem described by Equation 1 is formulated as

$$
\left\{\hat{\varphi}_{i}, \hat{I}\right\}_{i=1 \ldots N}=\underset{v_{i}: \dot{\phi}(t)=v\left(\phi^{v}, t\right), I}{\operatorname{argmin}} \sum_{i=1}^{N}\left(E^{2}\left(I, I_{i} \circ \varphi_{i}\right)+\int_{0}^{1}\left\|L v_{i}(t)\right\|_{V}^{2} d t\right) .
$$

Throughout this paper we use the square error dissimilarity metric. Under this metric, the template estimation problem becomes

$$
\begin{aligned}
\left\{\hat{\varphi}_{i}, \hat{I}\right\}_{i=1 \ldots N}= & \underset{v_{i}: \dot{\phi}(t)=v\left(\phi^{v}, t\right), I}{\operatorname{argmin}} \\
& \sum_{i=1}^{N}\left(\frac{1}{\sigma^{2}}\left\|I-I_{i} \circ \varphi_{i}\right\|_{L^{2}}^{2}+\int_{0}^{1}\left\|L v_{i}(t)\right\|_{V}^{2} d t\right)
\end{aligned}
$$

where $\sigma$ models the noise in the image match term. Smaller values of this parameter increase the penalty of image mismatch leading to exact matching when $\sigma \rightarrow 0$; this comes at the expense of smoothness in the estimated maps $\varphi_{i}$.

This minimization problem can be simplified by noticing that for fixed transformations $\varphi_{i}$, the image $\hat{I}$ that minimizes Equation 2 is given by the voxel-wise arithmetic mean of the deformed images

$$
\hat{I}=\frac{1}{N} \sum_{i=1}^{N} I_{i} \circ \varphi_{i}
$$


Combining Equations 2 and 3 results in following optimization, in terms of velocity fields,

$$
\begin{aligned}
\left\{\hat{v}_{i}\right\}_{i=1 \ldots N}= & \underset{\substack{v_{i}: \dot{\phi}(t)=v\left(\phi^{v}, t\right)\\
}}{\operatorname{argmin}} E\left(v_{i}\right) \doteq \\
& \sum_{i=1}^{N}\left(\frac{1}{\sigma^{2}}\left\|\left(\frac{1}{N} \sum_{j=1}^{N} I_{j} \circ \varphi_{j}\right)-I_{i} \circ \varphi_{i}\right\|_{L^{2}}^{2}+\int_{0}^{1}\left\|L v_{i}(t)\right\|_{V}^{2} d t\right)(4)
\end{aligned}
$$

For each individual velocity field, the minimizer of Equation 4 is constant speed, that is, $\left\|v_{i}(t)\right\|_{V}=c_{i}$, since it is a geodesic. Note that the solution to this minimization problem is independent of the ordering of the $N$ images.

\section{Implementation}

We use the full space time strategy presented in 13 . Since the minimization problem is independent of the ordering of the $N$ images we use an algorithm that estimates, on a per iteration basis, each $\varphi_{i}$ in turn. The optimization described by Equation 4 is implemented by an iterative steepest descent algorithm given by

$$
v_{i}^{k+1}(t)=v_{i}^{k}(t)-\epsilon \nabla_{v_{i}^{k}(t)} E
$$

where $\nabla E$ is the Gâteaux differential of the energy of the objective function giving the Euler-Lagrange condition. The Euler-Lagrange equation for the solution of the variational problem in 4 in space of smooth velocity fields $V$, becomes

$$
v_{i}(t)=\frac{1}{\sigma^{2}} K\left(\left|D \phi_{i}^{v}(t, 1)\right|\left(I \circ \phi_{i}^{v}(t, 0)-I_{i} \circ \phi_{i}^{v}(t, 1)\right) \nabla\left(I \circ \phi_{i}^{v}(t, 0)\right)\right)
$$

where $\phi_{i}(s, t)=\phi_{i}(t) \circ\left(\phi_{i}(s)\right)^{-1}$ and $I=\frac{1}{N} \sum_{j=1}^{N} I_{j} \circ \varphi_{j}$. The operator $K$ is the Green's function of the differential operator $L^{\dagger} L$ used to define the norm $\|\cdot\|_{V}$. In our implementation, $L$ is a modified Navier-Stokes operator [10]. Note that the stable point of Equation [6 satisfies the Euler-Lagrange equation $\nabla E=0$.

\subsection{Constant Speed Velocity}

The optimal velocity fields $\hat{v}_{i}$, given by Equation 4 , each define a geodesic path on the space of diffeomorphisms. As geodesics have constant speed, the velocity fields have constant norm over time. Given $\nabla E$, we enforce this geodesic constraint by calculating adaptive per-iteration time steps $\epsilon_{i}^{k}(t)$ such that for all time steps $t$, the norms $\int_{\Omega}\left\|L v_{i}^{k}(t)\right\|_{2}^{2} d x$ are equal. We formulate the inductive hypothesis: $\int_{\Omega}\left\|L v_{i}^{k}(t)\right\|_{2}^{2} d x=\rho_{i}^{k}$, a constant and solve for $\epsilon_{i}^{k+1}(t)$ such that

$$
\begin{aligned}
\int_{\Omega}\left\|L v_{i}^{k+1}\right\|_{2}^{2} d x & =\int_{\Omega}\left\|L\left[v_{i}^{k}-\epsilon_{i}^{k+1}(t) \nabla_{v_{t}^{k+1}} E\right]\right\|_{2}^{2} d x=\rho_{i}^{k+1} \\
\Rightarrow \rho_{i}^{k+1} & =\rho_{i}^{k}(t)-4 \lambda_{i}^{k+1}(t) \epsilon_{i}^{k+1}(t)+4 \nu_{i}^{k+1}(t)\left(\epsilon_{i}^{k+1}(t)\right)^{2}
\end{aligned}
$$


where

$$
\begin{aligned}
& \nu_{i}^{k+1}(t)=\rho_{i}^{k}-\frac{2}{\sigma^{2}} \int_{\Omega}\left\langle v_{i}^{k}(t), b_{i}^{k+1}(t)\right\rangle d x+\frac{1}{\sigma^{4}} \int_{\Omega}\left\langle b_{i}^{k+1}(t), K\left(b_{i}^{k+1}(t)\right)\right\rangle d x \\
& \lambda_{i}^{k+1}(t)=\rho_{i}^{k}-\frac{1}{\sigma^{2}} \int_{\Omega}\left\langle v_{i}^{k}(t), b_{i}^{k+1}(t)\right\rangle d x \\
& b_{i}^{k+1}(t)=\left|D \phi_{i}^{v}(t, 1)\right|\left(I \circ \phi_{i}^{v}(t, 0)-I_{i} \circ \phi_{i}^{v}(t, 1)\right) \nabla\left(I \circ \phi_{i}^{v}(t, 0)\right) .
\end{aligned}
$$

After choosing an appropriate value for the difference $\rho_{i}^{k+1}-\rho_{i}^{k}$, we solve Equation 7 for $\epsilon_{i}^{k+1}(t)$ using the quadratic formula for the positive solution which yields,

$$
\epsilon_{i}^{k+1}(t)=\frac{1}{2 \nu_{i}^{k+1}}\left(\lambda_{i}^{k+1}(t)+\sqrt{\left(\lambda_{i}^{k+1}(t)\right)^{2}-\nu_{i}^{k+1}(t)\left(\rho_{i}^{k}-\rho_{i}^{k+1}\right)}\right) .
$$

We begin by specifying an initial value for one of the $\epsilon_{i}^{k+1}(t)$ and solve for $\rho_{i}^{k+1}-\rho_{i}^{k}$. Note that the integrated velocity norm $\int_{\Omega}\left\|L v_{i}^{k+1}(t)\right\|_{2}^{2} d x$ does not have to be computed since it starts at zero and increases at iteration by $\rho_{i}^{k+1}-\rho_{i}^{k}$, which is known at each iteration.

\section{Results}

To evaluate the performance of this method, we consider the question: how many images are required to represent a population? To address this question, we build atlases of increasing population size and analyze their stability with respect to image intensity entropy. Entropy has often been proposed as a good measure of image quality [1415] where sharp images have relatively low entropy. Let $X$ be a random variable associated with the intensities for a given image and let $p_{X}$ be the probability mass function associated with $X$. Discrete entropy is defined as the expected uncertainty in $X$,

$$
H(X)=\mathcal{E}_{p_{X}}\left[-\log p_{X}(x)\right]=-\sum_{x \in X} p_{X}(x) \log p_{X}(x)
$$

where the logarithm, in our case, is take with base two yielding entropy measured in bits. The uniform distribution maximizes entropy for random variables defined without moment constraints, such as image intensities [17]. That is, a blurry image, with a relatively flat histogram, will have greater entropy than a sharp image. We are interested in the entropy introduced by the atlas creation method rather than the intrinsic entropy associated with images of individual brain anatomy.

\subsection{Atlas Formation}

Our image database contains fourteen brain images that have been provided by the UNC autism image analysis group. These images have been intensity normalized and rigidly aligned. Due to the high memory demands of our implementation, we apply our algorithm to 2D mid-axial slices, although the methodology is 
generalizable to 3D. These images are show in Figure1 There is noticeable large deformation variation between these anatomies, especially in lateral ventricles.

To quantify the stability of the estimated atlases, we generate eleven atlas cohorts, $\left\{C_{l}\right\}_{l=2 \ldots 12}$, each with twenty atlases derived from $l$ images randomly

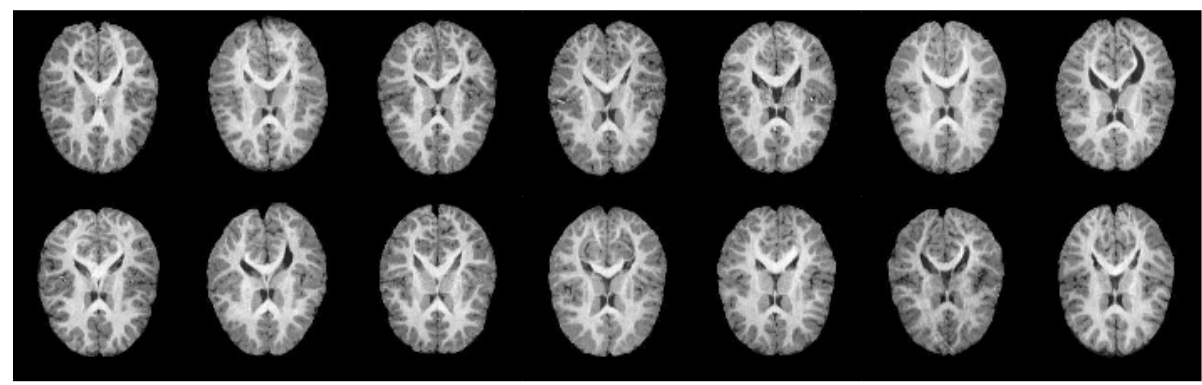

Fig. 1. Image Database: 2D mid-axial slices from MR images of fourteen subjects

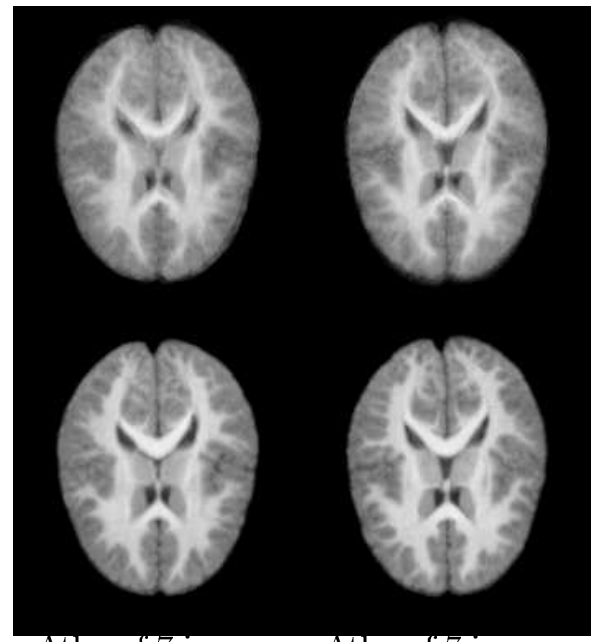

Atlas of 7 images Atlas of 7 images

Fig. 2. Mutually Exclusive Atlases: each column represents an individual atlas constructed by both arithmetically averaging rigidly aligned images (top row) and estimating a Fréchet mean atlas after 100 iterations (bottom row). These two atlases were formed from completely separate sets of images.

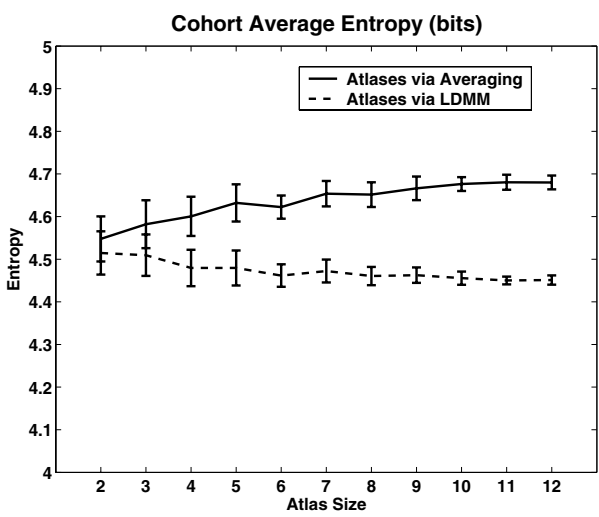

Fig. 3. Average Entropy: for comparison, the average entropy of the original fourteen images is 3.91 bits with standard deviation 0.08 bits. The error bars represent one standard deviation from the mean. Prior to computing entropy, we shift all atlases by half a pixel to compensate for the entropy introduced by the linear interpolation used to reconstruct images during registration. 
selected from the original database of fourteen images. Two mutually exclusive atlases from $C_{7}$ are shown in Figure 2 for both simple averaging and the LDMM method. The rigidly aligned atlases are blurry since they are arithmetic averages of varying individual neuroanatomies. Ghosting is evident around the lateral ventricles and near the boundary of the brain. In the final Fréchet atlases, these regions appear much sharper.

\subsection{Atlas Convergence}

To evaluate the robustness and stability of our atlases we first compute the mean and standard deviation of the entropies of the original fourteen images. To this we compare the mean and standard deviation of the atlas cohort entropies that have been created both by simple arithmetic averaging of the rigidly aligned images and those produced by the LDMM method. These results are summarized in Figure 3 From this plot we notice that as atlas size increases, the average atlas entropy increases for atlases formed by simple intensity averaging, where as the average entropy decreases for atlases created via LDMM. The atlases also become more stable with respect to entropy as the standard deviation decreases with atlas size. After cohort $C_{10}$, the atlas entropy means appear to converge. To answer our original question, given these fourteen subjects, we need about ten images to create a stable atlas representing neuroanatomy.

\section{Conclusion}

A novel method for unbiased atlas formation involving large deformations metric mapping has been presented. The LDMM implementation has also been improved by new constant speed velocity reparameterization constraint. The preliminary results show that this method produces stable atlases with respect to the entropy image quality measure. A possible direction for future work is to explore the stability and robustness in the presence of much larger initial population databases.

\section{Acknowledgments}

This work was supported by NIBIB-NIH grant R01 EB000219, NIH-HLB grant R01 HL69808, DOD Prostate Cancer Research Program DAMD17-03-1-0134, NIMH grant MH064580 Longitudinal MRI Study of Brain Development in Fragile X, and the NDRC.

\section{References}

1. Grenander, U., Miller, M.: Computational anatomy: an emerging discipline. Quarterly of Applied Mathematics 56 (1998) 617-694

2. Toga, A.W.: An Introduction to Brain Warping. Academic Press, San Diego (1999) 
3. Gee, J.C., Reivich, M., Bajcsy, R.: Elastically deforming an atlas to match anatomical brain images. Journal of Computer Assisted Tomography 17 (1993) 225-236

4. Miller, M.I., Younes, L.: Group actions, homeomorphisms, and matching: A general framework. Internation Journal of Computer Vision (IJCV) 41 (2001) 61-84

5. Rohlfing, T., Russakoff, D.B., Calvin R. Mauerer, J.: Extraction and application of expert priors to combine multiple segmentations of human brain tissue. In: Proceedings of Medical Image Computing and Computer-Assisted Intervention (MICCAI). Volume 2732., Springer-Verlag (2003) 578-585

6. Thompson, P.M., Toga, A.W.: A framework for computational anatomy. Computing and Visualization in Science 5 (2002) 13-34

7. Avants, B., Gee, J.C.: Geodesic estimation for large deformation anatomical shape averaging and interpolation. NeuroImage Supplement 1 (2004) S139-S150

8. Bhatia, K.K., Hajnal, J.V., Puri, B.K., Edwards, A.D., Ruekert, D.: Consistent groupwise non-rigid registration for atlas construction. In: Proceedings of IEEE International Symposium on Biomedical Imaging (ISBI). (2004) 908-911

9. Guimond, A., Meunier, J., Thirion, J.P.: Average brain models: a convergence study. Computer Vision and Image Understanding 77 (2000) 192-210

10. Joshi, S., Miller, M.I.: Landmark matching via large deformation diffeomorphisms. IEEE Transactions on Image Processing (TIP) 9 (2000) 1357-1370

11. Joshi, S., Davis, B., Jomier, M., Gerig, G.: Unbiased diffeomorphic atlas construction for computational anatomy. NeuroImage: Supplement issue on Mathematics in Brain Imaging 23 (2004) S151-S160

12. Fréchet, M.: Les elements aleatoires de nature quelconque dans un espace distancie. Annales De L'Institut Henri Poincare 10 (1948) 215-310

13. Beg, M.F.: Variational and Computational Methods for Flows of Diffeomorphisms in Image Matching and Growth in Computational Anatomy. PhD thesis, The Johns Hopkins University (2003)

14. Guillemaud, R., Brady, M.: Estimating the bias field of MR images. IEEE Transactions on Medical Imaging (TMI) 16 (1997) 878-886

15. Atkinson, D., Hill, D.L.G., Stoyle, P.N.R., Summers, P.E., Keevil, S.F.: Automatic correction of motion artifacts in magnetic resonance images using an entropy focus criterion. IEEE Transactions on Medical Imaging (TMI) 16 (1997) 903-910

16. Twining, C., Marsland, S., Taylor, C.: Measuring geodesic distances on the space of bounded diffeomorphisms. In: Proceedings of the British Machine Vision Conference (BMVC), BVMA Press (2002) 847-856

17. Cover, T., Thomas, J.: Elements of Information Theory. John Wiley and Sons, Inc., New York (1991) 